\title{
Immunomodulation by ketamine as an adjunct to total intravenous anesthesia in patients undergoing minimally invasive radical prostatectomy: A randomized pilot trial
}

\author{
JUN KAWAGUCHI ${ }^{1}$, DAICHI OTA ${ }^{1}$, HIDETOMO NIWA ${ }^{1}$, YUKI SUGO ${ }^{1}$, \\ TETSUYA KUSHIKATA ${ }^{2}$ and KAZUYOSHI HIROTA ${ }^{2}$ \\ ${ }^{1}$ Department of Anesthesiology, Hirosaki University Hospital, Hirosaki, Aomori 036-8563; ${ }^{2}$ Department of Anesthesiology, \\ Hirosaki University Graduate School of Medicine, Hirosaki, Aomori 036-8562, Japan
}

Received November 4, 2019; Accepted March 31, 2020

DOI: $10.3892 / \mathrm{mco} .2020 .2060$

\begin{abstract}
Post-surgery immunomodulation, including reduced natural killer cell cytotoxicity (NKCC), is recognized as a predictor of poor outcomes in patients following cancer surgery. The present study investigated direct immunomodulation via ketamine as an anesthetic adjuvant in patients undergoing cancer surgery. The present non-double blinded randomized trial was conducted at Hirosaki University Hospital with 60 patients who underwent minimally invasive robotic radical prostatectomy to minimize the immunomodulation due to surgical stress. Patients received total intravenous anesthesia using propofol and remifentanil, with ketamine as an anesthetic adjuvant (the ketamine group) or without ketamine (the control group). The primary outcome was the difference in NKCC between these groups. The secondary outcomes were the differences in neutrophil-lymphocyte ratio (NLR) and levels of interleukin (IL)-6, IL-1 $\beta$, IL-10 and tumor necrosis factor-alpha (TNF- $\alpha$ ). NKCC and cytokines were measured before anesthesia (baseline) and at 6 and $24 \mathrm{~h}$ after baseline measurements were recorded. NLR was determined on the last day before admission and at $48 \mathrm{~h}$ post-baseline. NKCC values were similar in each group at $6 \mathrm{~h}$ when compared with respective baseline results (baseline control, 36.9 $15.6 \%$; $6 \mathrm{~h}$ control,
\end{abstract}

Correspondence to: $\mathrm{Dr}$ Hidetomo Niwa, Department of Anesthesiology, Hirosaki University Hospital, 53 Honcho, Hirosaki, Aomori 036-8563, Japan

E-mail: niwahide@gmail.com

Abbreviations: BIS, bispectral index; CLEIA, chemiluminescent enzyme immunoassay; cpm, counts per minute; $\mathrm{Cr}$, chromium; EASIA, enzyme-amplified sensitivity immunoassay; IL, interleukin; MAP, mean arterial pressure; NK cells, natural killer cells; NKCC, natural killer cell cytotoxicity; NRS, numerical rating scale; TIVA, total intravenous anesthesia; TNF, tumor necrosis factor

Key words: ketamine, natural killer cell cytotoxicity, neutrophil-lymphocyte ratio, proinflammatory cytokine, direct immunomodulation, prostate cancer, robotic radical prostatectomy
$38.3 \pm 13.4 \%$; baseline ketamine, $36.1 \pm 17.0 \%$; 6 h ketamine, $36.6 \pm 16.4 \%$ ) but significantly decreased at $24 \mathrm{~h}$ (control, $26.5 \pm 12.2 \%$; ketamine, $24.1 \pm 12.7 \%$; $\mathrm{P}<0.001)$. There were no significant differences in NKCC between the ketamine and control groups $(\mathrm{P}=0.64)$ at any of the assessed time points. NLR, IL-1 $\beta$, IL-10 and TNF- $\alpha$ levels were also similar between two groups. In contrast, IL-6 at $24 \mathrm{~h}$ was significantly lower in the ketamine group compared with the control group (mean difference, - $7.3 \mathrm{pg} \mathrm{ml}^{-1} ; 95 \%$ confidence interval, -14.4 to $-0.2 ; \mathrm{P}=0.04)$. Ketamine as an anesthetic adjuvant did not provide direct immunomodulation in patients who underwent cancer surgery.

\section{Introduction}

Immunomodulation due to the body's stress response to surgical trauma such as reduced natural killer cell cytotoxicity (NKCC), an increased neutrophil-lymphocyte ratio (NLR), and elevated inflammatory cytokine secretion is recognized as a biological marker for predicting the poor outcome of cancer surgery $(1,2)$. It has been hypothesized that the choice of anesthesia to be used for cancer patients could potentially affect the risks of recurrence and metastases, because anesthetic agents can influence the body's stress response to surgical trauma, with subsequent immunomodulation due to the surgical stress (1,3-7). Some multicenter randomized clinical trials are being conducted to test this hypothesis.

In contrast, it has also been hypothesized that anesthetic agents could modulate immune cells directly and affect the outcome of cancer surgery (8). Many commonly used opioids have shown to modulate immune cells directly via the activation of the $\mu$ opioid receptor and non-opioid toll-like receptors. The most common opioid, morphine was reported to decrease NKCC and increase caner development (8) Accumulating evidence also shows that an intravenous sedative-hypnotic agent, propofol has direct effects via receptors present on natural killer cells (9).

Ketamine, the N-Methyl-D-aspartate receptor antagonist is an old anesthetic agent intravenously or intramuscularly given to human. In our institution, ketamine has been used for cancer or non-cancer surgery as an adjunct to total 
intravenous anesthesia (TIVA) with propofol and opioids since 1990 s, because this agent is expected to provide beneficial non-anesthetic effects for surgical patients $(10,11)$. However, the direct effect of ketamine on immune cells has not been well documented.

We thus conducted the present randomized clinical trial to determine whether ketamine used as an anesthetic adjuvant directly modulates immune cells of patients undergoing cancer surgery. This was a preliminary trial, primarily for patients undergoing minimally invasive prostate cancer surgery.

\section{Patients and methods}

Patient selection. This study was approved by the Hirosaki University Graduate School of Medicine Institutional Review Board (approval no. 2015-205), and written informed consent was obtained from all patients participating in the trial. Prior to the patients' enrollment, the trial was registered at the University Hospital Medical Information Network (registration no. UMIN000021231, Principal investigator: Hidetomo Niwa, Date of registration: February 28th, 2016). This prospective, randomized controlled study was carried out at Hirosaki University Hospital (Hirosaki, Japan).

Consecutive patients who underwent a robot-assisted radical prostatectomy were randomly assigned in the trial with the use of a computer-generated table to receive ketamine treatment (the ketamine group) or no ketamine treatment (the control group). We carried out a block randomization with two sets of blocks of two random combinations (ketamine and no ketamine treatment). HN generated the random allocation sequence. JK enrolled the participants and assigned the participants to interventions. The candidates for inclusion in the study were patients aged $\geq 18$ years whose American Society of Anesthesiologists (ASA) physical status was I-III. Patients who had an acute medical disease, a cognitive disorder, or a history of other cancer treatment within the prior 1 year, and urgent cases were not enrolled. Only minimally invasive surgery cases were enrolled, in order to minimize and standardize the patients' stress response to surgical trauma followed by their immunomodulation due to the surgical stress. The patients and the investigators measuring the patients' laboratory data were blinded after the patients' assignment to interventions; other investigators such as the attending anesthesiologists were not blinded.

\section{The trial protocol}

Anesthesia. The anesthesia protocol was standardized according to the drugs used. The patients in the ketamine group received TIVA using propofol and remifentanil with ketamine as described below. The patients in the control group received propofol and remifentanil anesthesia as described below. In light of the ethical concerns, a placebo was not used in the control group, and thus the attending anesthesiologists could not be blinded in this trial.

Anesthesia was induced with propofol (0.5-1.0 mg kg-1) and remifentanil (0.1-0.5 $\left.\mu \mathrm{g} \mathrm{kg}^{-1} \mathrm{~min}^{-1}\right)$ with/without ketamine $\left(1 \mathrm{mg} \mathrm{kg} \mathrm{kg}^{-1}\right)$, plus rocuronium $\left(0.6 \mathrm{mg} \mathrm{kg}^{-1}\right)$. After tracheal intubation, each attending anesthesiologist titrated the propofol and remifentanil over a dose of 3-7 $\mathrm{mg} \mathrm{kg}^{-1} \mathrm{~h}^{-1}$ for the propofol and $0.05-0.5 \mu \mathrm{g} \mathrm{kg}^{-1} \mathrm{~min}^{-1}$ for the remifentanil with electroencephalography-guided administration using a target bispectral index (BIS) value of approximately 40-60 (BIS-XP ${ }^{\circledR}$ system; Aspect Medical Systems) to maintain the patient's mean arterial pressure (MAP) and heart rate in a clinically acceptable range. Monitoring the BIS enables the tracking of the electroencephalogram changes associated with the changes in the depth of anesthesia.

Muscle relaxation was maintained with additional doses of rocuronium at $0.1 \mathrm{mg} \mathrm{kg}^{-1}$. In the ketamine group, ketamine was administered at $1 \mathrm{mg} \mathrm{kg}^{-1}$ for anesthetic induction and then continuously at $0.3 \mathrm{mg} \mathrm{kg}^{-1} \mathrm{~h}^{-1}$ in accord with our clinical practice. Standard monitoring including ECG, BIS, and pulse oximetry was used upon the patient's arrival in the operative suite. After the induction of anesthesia, a radial artery was cannulated for direct arterial blood pressure measurement.

Post-operative pain management. When the patient was placed back in the supine position and the surgeon began to close the incision, a bolus infusion of fentanyl $\left(4 \mu \mathrm{g} \mathrm{kg}^{-1}\right)$ was given. This was followed in the recovery suite by the use of intravenous patient-controlled analgesia (a basal infusion $0.25 \mu \mathrm{g} \mathrm{kg}^{-1} \mathrm{~h}^{-1}$ of fentanyl, a 2-ml bolus, and a lock-out time of $30 \mathrm{~min})$. A dose of $10 \mathrm{mg} \mathrm{kg}^{-1}$ of intravenous acetaminophen was also given every $6 \mathrm{~h}$ post-surgery.

\section{Measurements}

The patients' demographic data. The following demographic and clinical data were collected: Age, height, body weight, and the ASA physical status classification. Each patient's surgical data including the total dose of each anesthetic agent used, the pain intensity after surgery (on a numerical rating scale, NRS), and the duration of surgery/anesthesia were also collected.

The NKCC measurement. We measured each patient's NKCC by performing a $3.5 \mathrm{~h}$ chromium $-51\left({ }^{51} \mathrm{Cr}\right)$ release assay using the reaction of peripheral blood mononuclear cells (monocytes and lymphocytes) and ${ }^{51} \mathrm{Cr}$-labeled $\mathrm{K} 562$ cells at an effector/target ratio of 20:1. The NKCC was calculated according to the following formula: NKCC $(\%)=$ experimental release (counts per minute, $\mathrm{cpm}$ )-spontaneous release (cpm) $[\max . \text { release }(\mathrm{cpm}) \text {-spontaneous release }(\mathrm{cpm})]^{-1} \mathrm{x} 100$.

The NLR. We calculated each patient's NLR (neutrophil lymphocyte count ${ }^{-1}$ ) using the patient's pre- and post-operative laboratory data. The pre-operative laboratory data were collected on the last day before the patient's admission, and the post-operative data were collected at $48 \mathrm{~h}$ after the induction of anesthesia.

The serum interleukin (IL)-1 $\beta,-6$, and -10 and TNF- $\alpha$ measurements. The patients' serum IL-1 $\beta,-10$, and tumor necrosis factor-alpha (TNF- $\alpha$ ) levels were measured by enzyme-linked immunosorbent assays (ELISAs) according to each manufacturer's instructions. Briefly, blood was collected at each time point, allowed to clot at room temperature for $30 \mathrm{~min}$, and then centrifuged for $10 \mathrm{~min}\left(4^{\circ} \mathrm{C}, 1000\right.$ rotations per minute). The serum samples were quickly frozen at $-20^{\circ} \mathrm{C}$ and stored until the day of analysis. The serum IL-1 $\beta$ levels were measured using a Biosource IL-1 $\beta$ EASIA kit 
(enzyme-amplified sensitivity immunoassay, serum IL-10 levels were measured using a Biosource IL-10 EASIA kit (both from BioSource Europe SA).

The serum TNF- $\alpha$ was measured using a quantitative high-sensitivity sandwich immunoassay (Quantikine ${ }^{\circledR} \mathrm{HS}$ Human TNF- $\alpha /$ TNFSF1A Immunoassay) from R\&D Systems. The serum IL- 6 was measured by a chemiluminescent enzyme immunoassay (CLEIA) using a Human IL-6 CLEIA Fujirebio kit (Fujirebio Inc.). The investigators measuring these laboratory data were blind to the patients' group allocations.

Primary and secondary outcome measures. The primary outcome was the difference in NKCC between the ketamine and control groups. The secondary outcomes were the difference in the NLR and cancer progression-related cytokines, i.e., serum IL-1 $\beta,-6$, and -10 and TNF- $\alpha$. Blood sampling for the measurement of the NKCC and cytokines was conducted before the administration of anesthesia ( $0 \mathrm{~h}$, i.e., baseline), at 6 and at $24 \mathrm{~h}$ after the induction of the anesthesia.

Statistical analysis. For continuous variables with a normal distribution, the mean \pm standard deviation (SD) or standard error (SE) is reported. For variables not normally distributed, the median and interquartile ranges are reported. $\mathrm{P}<0.05$ were considered significant. The $\chi^{2}$ test was used for the analysis of categorical data, and Student's t-test was used for continuous variables with normal distributions. The Mann-Whitney rank-sum test was used for continuous variables without a normal distribution. We performed a repeated-measures analysis of variance (ANOVA) with a Bonferroni correction to determine the differences in NKCC, IL-1 $\beta,-6$, and -10 and TNF- $\alpha$ between the ketamine and control groups.

Sample size calculations were performed using G*Power 3 software (10). We performed a power analysis by using a repeated measures ANOVA with an effect size of 0.25 . Bentley et al (11) suggested that an effect size of 0.25 is a medium effect for the ANOVA statistic. A power analysis with an effect size of 0.25 for the power of 0.80 at a two-sided alpha level of 0.05 showed that it was necessary to have $\geq 29$ subjects in each group. All statistical analyses were conducted with IBM SPSS ${ }^{\circledR}$ statistics ver. 22.0 software (IBM Corp.).

\section{Results}

A total of 67 patients treated during the period from April 2017 to March 2018 were included in the analyses. A flow diagram of the patients is given in Fig. 1. One patient in the ketamine group was excluded because he had an unexpected injury in the small intestine that needed to be repaired during surgery. Another patient in the control group was excluded because of a missed blood sampling. Five patients were excluded because they received extra analgesics during or after surgery that may have affected the study's primary outcome.

The demographics of the patients and the details of their anesthesia are summarized in Table I. All clinical characteristics were comparable between the ketamine and control groups except for the post-operative pain intensity and the doses of propofol and remifentanil used during the surgery. The doses of propofol and remifentanil used were significantly higher in the ketamine group compared to the control group. In contrast,

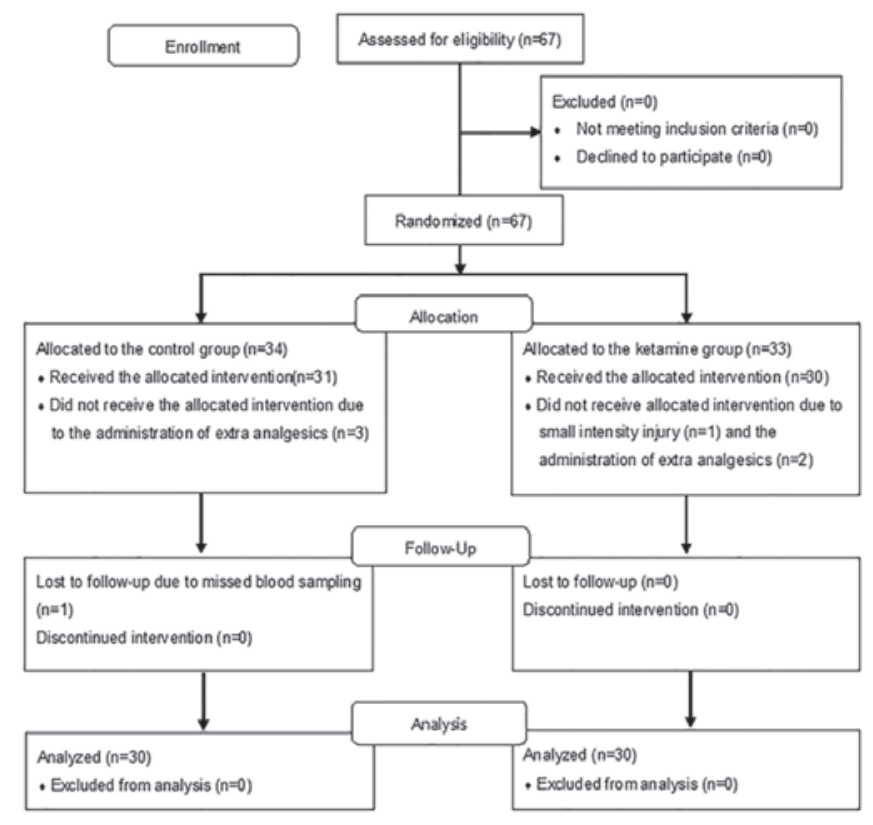

Figure 1. Flow diagram of the patients included in the present study.

the post-operative pain intensity evaluated using the NRS was significantly lower in the ketamine group. However, the difference in the median NRS values between the two groups was not a clinically important value as it was quite small: Median $\mathrm{NRS}=1$ [interquartile range $(\mathrm{IQR}) 0,3$ ] in the control group, and $0(0,2.8)$ in the ketamine group. No harm or unintended events related to this trial were identified.

The NKCC. The NKCC in the ketamine group changed with essentially the same time course as that of the control group, as follows: Compared to each baseline value of NKCC, each group's NKCC was comparable to baseline at $6 \mathrm{~h}$ but significantly decreased at $24 \mathrm{~h}$ after the anesthesia induction $(\mathrm{P}<0.001$, Table II). That is, we found no significant difference in NKCC between the ketamine and the control groups at any of the time points $(\mathrm{P}=0.64$, Table II).

The NLR and the serum values of $I L-1 \beta,-6$, and -10 and $T N F-\alpha$. As shown in Table II, we also observed no significant between-group difference in the changes in the NLR after surgery $(\mathrm{P}=0.9)$. Serum IL-1 $\beta$ and IL-10 were not detected at any of the three time points in the ketamine group or in the control group. The level of serum TNF- $\alpha$ in was similar in the two groups at all time points. In contrast, the serum IL-6 levels in both groups were significantly increased at $6 \mathrm{~h}$ after the induction of anesthesia with a peak value $(\mathrm{P}<0.001)$ and then slightly decreased at $24 \mathrm{~h}$, but the levels were still significantly higher than those obtained at baseline $(\mathrm{P}<0.001)$. At $24 \mathrm{~h}$ after the induction of anesthesia, the serum IL-6 levels of the ketamine group were significantly lower than those of the control group $(\mathrm{P}=0.04)$.

\section{Discussion}

In this single-blinded randomized trial, we tested whether the use of ketamine would directly modulate immune cells 
Table I. Characteristics and surgical details of the control and ketamine groups.

\begin{tabular}{|c|c|c|c|c|}
\hline Parameter & Control $(n=30)$ & Ketamine $(\mathrm{n}=30)$ & Mean difference $(95 \% \mathrm{CI})$ & P-value \\
\hline Age (years) & $68.7 \pm 5.7$ & $67.1 \pm 6.1$ & $-1.5(-4.6$ to 1.5$)$ & 0.32 \\
\hline Height $(\mathrm{cm})$ & $165.9 \pm 6.0$ & $166.4 \pm 6.3$ & $0.5(-2.6$ to 3.7$)$ & 0.74 \\
\hline Weight (kg) & $66.0 \pm 9.1$ & $69.0 \pm 10.5$ & $3.0(-2.1$ to 8.1$)$ & 0.24 \\
\hline BMI & $23.9 \pm 2.7$ & $24.8 \pm 2.9$ & $0.9(-0.5$ to 2.4$)$ & 0.20 \\
\hline ASA & $2[2,2]$ & $2[2,2]$ & n.a. & 0.10 \\
\hline Anesthesia time (min) & $224 \pm 44$ & $235 \pm 37$ & 11 (-10 to 32$)$ & 0.30 \\
\hline Surgical period (min) & $158 \pm 44$ & $169 \pm 36$ & $11(-10$ to 31$)$ & 0.31 \\
\hline Propofol (mg) & $1,055 \pm 296$ & $1,285 \pm 407^{\mathrm{a}}$ & $230(45$ to 414$)$ & 0.02 \\
\hline Remifentanil $(\mu \mathrm{g})$ & $2,183 \pm 717$ & $2,808 \pm 890^{b}$ & $626(208$ to 1044$)$ & $>0.001$ \\
\hline Ketamine (mg) & 0 & $137 \pm 25^{\mathrm{c}}$ & $-137(-146$ to -129$)$ & $>0.001$ \\
\hline Fentanyl $(\mu \mathrm{g})$ & $265 \pm 52$ & $272 \pm 47$ & 7 (19 to 32$)$ & 0.61 \\
\hline Acetaminophen (mg) & $805 \pm 165$ & $846 \pm 184$ & $41(-49$ to 131$)$ & 0.37 \\
\hline IVPCA (fentanyl) $(\mu \mathrm{g})$ & $830 \pm 115$ & $867 \pm 136$ & 37 (-28 to 102$)$ & 0.26 \\
\hline Pain intensity (NRS) & $1[0,3]$ & $0[0,2.8]$ & n.a. & 0.05 \\
\hline
\end{tabular}

${ }^{\mathrm{a}} \mathrm{P}<0.05,{ }^{\mathrm{b}} \mathrm{P}<0.01$ and ${ }^{\mathrm{c}} \mathrm{P}<0.001$ (control vs. ketamine). Data are presented as the mean \pm standard deviation or median (minimal, maximum value). Mean difference, ketamine group-control group; CI, confidence interval; ASA, American Society of Anesthesiologists Physical Status Classification Scale; BMI, body mass index; IVPCA, intravenous patient-controlled analgesia; n.a., not analyzed; NRS, numerical rating scale.

Table II. The NKCC, NLR, TNF- $\alpha$, IL-6, IL-10 and IL-1 $\beta$ values of the control and ketamine groups at baseline at 6 and $24 \mathrm{~h}$ after the induction of anesthesia.

\begin{tabular}{|c|c|c|c|c|}
\hline Parameter & Control $(n=30)$ & Ketamine $(n=30)$ & Mean difference $(95 \% \mathrm{CI})$ & P-value \\
\hline $\mathrm{NKCC}$ at $0 \mathrm{~h}(\%)$ & $36.9 \pm 15.6$ & $36.1 \pm 17.0$ & $-0.8(-9.3$ to 7.7$)$ & 0.85 \\
\hline $\mathrm{NKCC}$ at $6 \mathrm{~h}(\%)$ & $38.3 \pm 13.4$ & $36.6 \pm 16.4$ & $-1.6(-9.3$ to 6.1$)$ & 0.68 \\
\hline NKCC at $24 \mathrm{~h}(\%)$ & $26.5 \pm 12.2^{\mathrm{a}}$ & $24.1 \pm 12.7^{\mathrm{a}}$ & $-2.3(-8.8$ to 4.1$)$ & 0.47 \\
\hline NLR before surgery & $3.3 \pm 2.0$ & $3.0 \pm 1.4$ & $-0.2(-1.1$ to 0.6$)$ & 0.59 \\
\hline NLR after surgery & $6.5 \pm 2.4$ & $6.2 \pm 3.7$ & $-0.3(-1.9$ to 1.3$)$ & 0.69 \\
\hline Delta NLR & $3.2 \pm 1.8$ & $3.1 \pm 3.4$ & $-0.1(-1.5$ to 1.3$)$ & 0.90 \\
\hline $\mathrm{TNF}-\alpha$ at $0 \mathrm{~h}\left(\mathrm{pg} \mathrm{ml} \mathrm{l}^{-1}\right)$ & $1.0 \pm 0.5$ & $1.0 \pm 0.4$ & $0(-0.2$ to 0.2$)$ & 0.96 \\
\hline 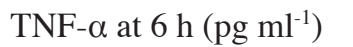 & $1.0 \pm 0.5$ & $1.1 \pm 0.5$ & $0.1(-0.2$ to 0.3$)$ & 0.68 \\
\hline TNF- $\alpha$ at $24 \mathrm{~h}\left(\mathrm{pg} \mathrm{ml} \mathrm{m}^{-1}\right)$ & $1.1 \pm 0.5$ & $1.1 \pm 0.4$ & $0.1(-0.2$ to 0.3$)$ & 0.67 \\
\hline IL-6 at $0 \mathrm{~h}\left(\mathrm{pg} \mathrm{ml}^{-1}\right)$ & $1.5 \pm 1.0$ & $1.3 \pm 0.8$ & $-0.2(-0.6$ to 0.3$)$ & 0.42 \\
\hline IL-6 at $6 \mathrm{~h}\left(\mathrm{pg} \mathrm{ml} \mathrm{m}^{-1}\right)$ & $51.4 \pm 23.4^{\mathrm{a}}$ & $41.5 \pm 22.9^{\mathrm{a}}$ & $-10.0(-22.0$ to 2.0$)$ & 0.10 \\
\hline IL-6 at $24 \mathrm{~h}\left(\mathrm{pg} \mathrm{ml}^{-1}\right)$ & $34.4 \pm 13.9^{\mathrm{a}}$ & $27.1 \pm 13.5^{\mathrm{a}, \mathrm{b}}$ & $-7.3(-14.4$ to -0.2$)$ & 0.04 \\
\hline IL-10 at $0 \mathrm{~h}\left(\mathrm{pg} \mathrm{ml}^{-1}\right)$ & n.d. & n.d. & n.d. & n.a. \\
\hline IL-10 at $6 \mathrm{~h}\left(\mathrm{pg} \mathrm{ml}^{-1}\right)$ & n.d. & n.d. & n.d. & n.a. \\
\hline IL-10 at $24 \mathrm{~h}\left(\mathrm{pg} \mathrm{ml}^{-1}\right)$ & n.d. & n.d. & n.d. & n.a. \\
\hline $\mathrm{IL}-1 \beta$ at $0 \mathrm{~h}\left(\mathrm{pg} \mathrm{m}^{-1}\right)$ & n.d. & n.d. & n.d. & n.a. \\
\hline IL- $1 \beta$ at $6 \mathrm{~h}\left(\mathrm{pg} \mathrm{ml}^{-1}\right)$ & n.d. & n.d. & n.d. & n.a. \\
\hline IL-1 $\beta$ at $24 \mathrm{~h}\left(\mathrm{pg} \mathrm{ml}^{-1}\right)$ & n.d. & n.d. & n.d. & n.a. \\
\hline
\end{tabular}

${ }^{\mathrm{a}} \mathrm{P}<0.001,0$ vs. $6 \mathrm{~h}$ and $24 \mathrm{~h} .{ }^{\mathrm{b}} \mathrm{P}<0.05$ control vs. ketamine group. Data are the mean \pm standard deviation. Delta NLR, NLR value after surgery-before surgery; IL, interleukin; n.a., not analyzed; n.d., not detected; NKCC, natural killer cell cytotoxicity; NLR, neutrophil-lymphocyte ratio; TNF, tumor necrosis factor; TNF, tumor necrosis factor- $\alpha$; CI, confidence interval.

of patients who underwent a radical resection of prostate cancer. The results of our analyses demonstrated that ketamine administration as an adjunct to TIVA did not directly modulate immune cells when administered to patients undergoing cancer surgery, as the NKCC, the NLR, and the levels of inflammatory cytokines (except for IL-6) in the ketamine group changed after surgery in exactly the same manner as that observed in the control group. The results of our analyses 
also revealed that the increase in serum IL- 6 was significantly lower in the ketamine group. However, the difference in the groups' IL-6 levels was small; the mean difference between the ketamine and control groups was $-7.3 \mathrm{pg} \mathrm{ml}^{-1}$. Trials with much larger numbers of patients are thus necessary to reliably estimate the effects of ketamine on cytokine responses.

A methodological concern might be present in this trial; we investigated whether ketamine has a direct effect on patients immune cells, and our results indicated that it does not. We believe that this trial could have revealed such an effect, for the following reason: Indirect immunomodulation by anesthetic agents is due to agents' anti-nociceptive and anti-inflammatory action. Our findings demonstrated that the inhibition of the increase in IL- 6 as well as the decreased pain intensity after surgery were quite small in the ketamine group. These results indicate that indirect immunomodulation by ketamine due to its anti-inflammatory and anti-nociceptive actions could also be small and ignored in this trial. Only patients who underwent minimally invasive robotic surgery were enrolled in this trial. The single operative method that was used with minimal invasiveness may have helped minimize the variation in surgical invasiveness and standardize the patients' stress responses to surgical trauma, followed by inflammation that modulates immune cells.

Our findings are in disharmony with the hypothesis of previous studies in which ketamine was considered immunosuppressive $(12,13)$. Melamed and colleagues (14) demonstrated that Fischer rats anesthetized by ketamine showed reduced NKCC and increased lung metastases. Forget and colleagues (15) also showed that ketamine depressed the $\mathrm{NKCC}$ in non-operated rats. We speculate that the discrepancy between these prior findings and our present results is due to the difference in subjects (rats vs. humans).

Our findings are partially consistent with those of two randomized clinical studies $(11,16)$ which showed that ketamine administered before the surgery began did not affect the NKCC of patients undergoing abdominal (16) and oral maxillofacial (11) surgery. Despite this similarity in findings, we contend that our present findings provide different clinical information from that obtained in these two trials because the patients in those two trials underwent highly invasive surgery (i.e., abdominal hysterectomy, gastroplasty, and oral maxillofacial surgery). Those trials demonstrated that ketamine significantly inhibited the increase in inflammatory cytokines (16) and pain intensity (11) after the surgeries. Those results thus indicate that ketamine did not preserve the NKCC reduction caused by the surgical stress; i.e., the two trials investigated the indirect immunomodulation by ketamine.

Our present investigation is deemed to be fully powered due to the patients' homogeneity. Our patient series was only males who underwent a single operative method for prostate cancer, as this trial was preliminary. There was no variation of data due to gender or the cancer type. In contrast, the small number of patients in Beilin's study (16) $(n=39)$ were a 'non-cancer population' who underwent heterogenous surgeries. Bentley et al (11) enrolled 50 patients who were undergoing oral maxillofacial surgery, and they assigned the patients to three groups. In their clinical trial, each group was also divided by gender, and the authors compared the males versus females regarding each outcome. Their trial thus had six groups and was underpowered due to a small number of patients in each treatment group.

The present trial also has some limitations. First, it was a single-center, randomized clinical trial with only male patients who underwent surgery with a single operative method for prostate cancer. Our results should thus be interpreted cautiously when decision-making in clinical settings is considered. In addition, more remifentanil and propofol were used in the ketamine group than in the control group. We did have a standardized plan, but the exact dosing was at the discretion of the anesthesiologists (as it should be); propofol and remifentanil were titrated by each attending anesthesiologist to maintain the patients' MAP and heart rate values in a clinically acceptable range, with EEG guidance. We speculate that the higher BIS values were observed in the ketamine group because it has been reported that ketamine increases the BIS value $(17,18)$. Thus, some attending anesthesiologists may have concluded that lighter anesthesia was obtained in the patients in the ketamine group, and they thus administered more remifentanil and propofol against it. It is uncertain whether such non-standardized drug usage affected the results of this study.

Propofol has been shown to preserve or suppress NKCC to a lesser degree than any other anesthetics $(4,7)$. In contrast, the specific effects of remifentanil on NKCC are unclear due to the limited current literature related to remifentanil (19-21). A significant reduction of NKCC due to remifentanil infusion in a rat model was reported (21), whereas no alteration in the number or the cytotoxic function of NK cells was observed in healthy volunteers given low-dose remifentanil (19).

Finally, the natural response to surgical injury is composed of two phases. One phase known as hypometabolic period lasts for up to $12 \mathrm{~h}$ after surgical incision. Pro-inflammatory cytokine and stress hormones begin to increase during this period. Another phase is associated with hypermetabolism which persists for 7 days but occasionally lasts up to 3 weeks after surgery (22). During hypermetabolic phase, cellular immune function including NKCC and cytokine production are at its lowest by post-operative day (POD) 3 but gradually return to the baseline level by POD 7 (23). In the present study, NKCC and cytokine levels thus should've been measured at 24, 48, $72 \mathrm{~h}$, and 5 days after surgery. We are now investigating this point and intend to report in a later paper.

In conclusion, we performed a preliminary clinical trial to assess the direct effect of ketamine as an anesthetic adjuvant on patients' immune cells. Our results demonstrated that ketamine as an adjunct to TIVA did not modulate the immune cells directly when administered to patients undergoing a minimally invasive prostatectomy.

\section{Acknowledgements}

A part of this work was presented at the 25th Biennial Congress of the European Association for Cancer Research (Amsterdam) from June 30 to July 3, 2018.

\section{Funding}

This present study was supported by a Grant-in-Aid for Scientific Research (grant no. 16K20078) from the Japanese Society for the Promotion of Science (KAKENHI). 


\section{Availability of data and materials}

The datasets used and/or analyzed during the present study are available from the corresponding author on reasonable request.

\section{Authors' contributions}

JK and DO obtained patients written informed consent, administered clinical anesthesia and collected the data. HN designed the present study, collected and analyzed the data and wrote the manuscript. YS administered clinical anesthesia and collected the data. TK obtained patient written informed consent, collected the data and supervised the administration of clinical anesthesia. KH designed the present study, provided supervision and revised the manuscript for important intellectual content. All authors read and approved the final version of the manuscript.

\section{Ethics approval and consent to participate}

The present study was approved by the Hirosaki University Graduate School of Medicine Institutional Review Board (Hirosaki, Japan; approval no. 2015-205). Patients who participated in this research had complete clinical data. The signed informed consents were obtained from the patients or the guardians.

\section{Patient consent for publication}

Not applicable.

\section{Competing interests}

The authors declare that they have no competing interests.

\section{References}

1. Wall T, Sherwin A, Ma D and Buggy DJ: Influence of perioperative anaesthetic and analgesic interventions on oncological outcomes: A narrative review. Br J Anaesth 123: 135-150, 2019.

2. Ní Eochagáin A, Burns D, Riedel B, Sessler DI and Buggy DJ: The effect of anaesthetic technique during primary breast cancer surgery on neutrophil-lymphocyte ratio, platelet-lymphocyte ratio and return to intended oncological therapy. Anaesthesia 73 603-61, 2018.

3. Yoo S, Lee HB, Han W, Noh DY, Park SK, Kim WH and Kim JT: Total intravenous anesthesia versus inhalation anesthesia for breast cancer surgery: A retrospective cohort study. Anesthesiology 130: 31-40, 2019.

4. Sessler DI and Riedel B: Anesthesia and cancer recurrence: Context for divergent study outcomes. Anesthesiology 130: 3-5, 2019.

5. Forget P, Aguirre JA, Bencic I, Borgeat A, Cama A, Condron C, Eintrei C, Eroles P, Gupta A, Hales TG, et al: How anesthetic, analgesic and other non-surgical techniques during cancer surgery might affect postoperative oncologic outcomes: A summary of current state of evidence. Cancers (Basel) 11: pii: E592, 2019.
6. Pérez-González O, Cuéllar-Guzmán LF, Soliz J and Cata JP: Impact of regional anesthesia on recurrence, metastasis, and immune response in breast cancer surgery: A systematic review of the literature. Reg Anesth Pain Med 42: 751-756, 2017.

7. Wigmore TJ, Mohammed K and Jhanji S: Long-term survival for patients undergoing volatile versus IV anesthesia for cancer surgery: A retrospective analysis. Anesthesiology 124: 69-79, 2016.

8. Boland JW and Pockley AG: Influence of opioids on immune function in patients with cancer pain: From bench to bedside. Br J Pharmacol 175: 2726-2736, 2018.

9. Jiang S, Liu Y, Huang L, Zhang F and Kang R: Effects of propofol on cancer development and chemotherapy: Potential mechanisms. Eur J Pharmacol 831: 46-51, 2018.

10. Faul F, Erdfelder E, Buchner A and Lang AG: Statistical power analyses using $\mathrm{G}^{*}$ Power 3.1: Tests for correlation and regression analyses. Behav Res Methods 41: 1149-1160, 2009.

11. Bentley MW, Stas JM, Johnson JM, Viet BC and Garrett N: Effects of preincisional ketamine treatment on natural killer cell activity and postoperative pain management after oral maxillofacial surgery. AANA J 73: 427-436, 2005.

12. Liu FL, Chen TL and Chen RM: Mechanisms of ketamine-induced immunosuppression. Acta Anaesthesiol Taiwan 50: 172-177, 2012.

13. Heaney A and Buggy DJ: Can anaesthetic and analgesic techniques affect cancer recurrence or metastasis? Br J Anaesth 109 (Suppl 1): i17-i28, 2012.

14. Melamed R, Bar-Yosef S, Shakhar G, Shakhar K and Ben-Eliyahu S: Suppression of natural killer cell activity and promotion of tumor metastasis by ketamine, thiopental, and halothane, but not by propofol: Mediating mechanisms and prophylactic measures. Anesth Analg 97: 1331-1339, 2003.

15. Forget $\mathrm{P}$, Collet V, Lavand'homme $\mathrm{P}$ and De Kock M: Does analgesia and condition influence immunity after surgery? Effects of fentanyl, ketamine and clonidine on natural killer activity at different ages. Eur J Anaesthesiol 27: 233-240, 2010.

16. Beilin B, Rusabrov Y, Shapira Y, Roytblat L, Greemberg L, Yardeni IZ and Bessler H: Low-dose ketamine affects immune responses in humans during the early postoperative period. Br J Anaesth 99: 522-527, 2007.

17. Sengupta S, Ghosh S, Rudra A, Kumar P, Maitra G and Das T: Effect of ketamine on bispectral index during propofol-fentanyl anesthesia: A randomized controlled study. Middle East J Anaesthesiol 21: 391-395, 2011.

18. Phillips W, Anderson A, Rosengreen M, Johnson J and Halpin J: Propofol versus propofol/ketamine for brief painful procedures in the emergency department: Clinical and bispectral index scale comparison. J Pain Palliat Care Pharmacother 24: 349-355, 2010.

19. Cronin AJ, Aucutt-Walter NM, Budinetz T, Bonafide CP, DiVittore NA, Gordin V, Schuler HG and Bonneau RH: Low-dose remifentanil infusion does not impair natural killer cell function in healthy volunteers. Br J Anaesth 91: 805-809, 2003.

20. Moyano J and Aguirre L: Opioids in the immune system: From experimental studies to clinical practice. Rev Assoc Med Bras (1992) 65: 262-269, 2019.

21. Sacerdote P, Gaspani L, Rossoni G, Panerai AE and Bianchi M: Effect of the opioid remifentanil on cellular immune response in the rat. Int Immunopharmacol 1: 713-719, 2001.

22. Angka L, Khan ST, Kilgour MK, Xu R, Kennedy MA and Auer RC: Dysfunctional natural killer cells in the aftermath of cancer surgery. Int J Mol Sci 18: pii: E1787, 2017.

23. Bakos O, Lawson C, Rouleau S and Tai LH: Combining surgery and immunotherapy: Turning an immunosuppressive effect into a therapeutic opportunity. J Immunother Cancer 6: 86, 2018. 